\title{
Accounting for the effect of horizontal gradients in limb measurements of scattered sunlight
}

\author{
J. Puķīte ${ }^{1,2}$, S. Kühl ${ }^{1}$, T. Deutschmann ${ }^{3}$, U. Platt ${ }^{3}$, and T. Wagner ${ }^{1}$ \\ ${ }^{1}$ Max Planck Institute for Chemistry, J.J. Becher Weg 27, 55128 Mainz, Germany \\ ${ }^{2}$ Institute of Atomic Physics and Spectroscopy, University of Latvia, Raina Bulv. 19, Riga, 1586, Latvia \\ ${ }^{3}$ Institute of Environmental Physics, University of Heidelberg, Im Neuenheimer Feld 229, 69120 Heidelberg, Germany
}

Received: 12 October 2007 - Published in Atmos. Chem. Phys. Discuss.: 19 November 2007

Revised: 13 May 2008 - Accepted: 16 May 2008 - Published: 20 June 2008

\begin{abstract}
Limb measurements provided by the SCanning Imaging Absorption spectrometer for Atmospheric CHartographY (SCIAMACHY) on the ENVISAT satellite allow retrieving stratospheric profiles of various trace gases on a global scale, among them $\mathrm{BrO}$ for the first time. For limb observations in the UV/VIS spectral region the instrument measures scattered light with a complex distribution of light paths: the light is measured at different tangent heights and can be scattered or absorbed in the atmosphere or reflected by the ground. By means of spectroscopy and radiative transfer modelling these measurements can be inverted to retrieve the vertical distribution of stratospheric trace gases.
\end{abstract}

The fully spherical 3-D Monte Carlo radiative transfer model "Tracy-II" is applied in this study. The Monte Carlo method benefits from conceptual simplicity and allows realizing the concept of full spherical geometry of the atmosphere and also its 3-D properties, which is important for a realistic description of the limb geometry. Furthermore it allows accounting for horizontal gradients in the distribution of trace gases.

In this study the effect of horizontally inhomogeneous distributions of trace gases along flight/viewing direction on the retrieval of profiles is investigated. We introduce a tomographic method to correct for this effect by combining consecutive limb scanning sequences and utilizing the overlap in their measurement sensitivity regions. It is found that if horizontal inhomogenity is not properly accounted for, typical errors of $20 \%$ for $\mathrm{NO}_{2}$ and up to $50 \%$ for $\mathrm{OClO}$ around the altitude of the profile peak can arise for measurements close to the Arctic polar vortex boundary in boreal winter.

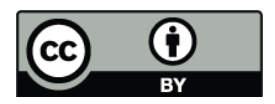

Correspondence to: J. Puķīe

(janis.pukite@mpch-mainz.mpg.de)

\section{Introduction}

While nadir observations (i.e. space borne instruments looking perpendicularly to the surface of the Earth) provide knowledge of the total column density and low vertical resolution information (about $10 \mathrm{~km}$ ) about profiles for strong absorbers only (Bhartia et al., 1996; Hoogen et al., 1999), measurements in limb geometry (i.e. tangential view with respect to the Earth surface) provide further opportunities to extract height resolved profile information. This is achieved by measuring backscattered light from air masses at different tangent heights. Satellite instruments such as the Optical Spectrograph and Infrared Imager System (OSIRIS) on the Odin satellite (Llewellyn et al., 2004), SCIAMACHY on ENVISAT (Bovensmann et al., 1999), and also the Stratospheric Aerosol and Gas Experiment (SAGE III) on the Meteor 3 have limb observation capabilities (Rault, 2005) in the UV/VIS spectral region.

The SCIAMACHY instrument on the ENVISAT satellite whose measurements are applied in this study operates in a near polar sun synchronous orbit with an inclination from the equatorial plane of $\sim 98.5^{\circ}$. It performs one orbit in approximately $100 \mathrm{~min}$ with equator crossing time of 10:00 in descending node. The satellite probes the atmosphere at the day side of Earth in alternating sequences of nadir and limb measurements. Limb scans in one scanning sequence are performed with approximately $3.3 \mathrm{~km}$ elevation steps at the tangent point (TP) in flight direction. The cross track swath is $960 \mathrm{~km}$ at the TP and consists of up to 4 pixels for the UV/VIS spectral range. The field of view (FOV) is $0.045^{\circ}$ in elevation and $1.8^{\circ}$ in azimuth. This corresponds to approximately $2.5 \mathrm{~km}$ in vertical direction and $110 \mathrm{~km}$ in horizontal direction at TP, respectively. SCIAMACHY measures in the UV-VIS-NIR spectral range from 240 to $2380 \mathrm{~nm}$ with a spectral resolution of approximately 0.25 to $0.55 \mathrm{~nm}$ in the UV-VIS range. More instrumental details can be found in Bovensmann et al. (1999).

Published by Copernicus Publications on behalf of the European Geosciences Union. 


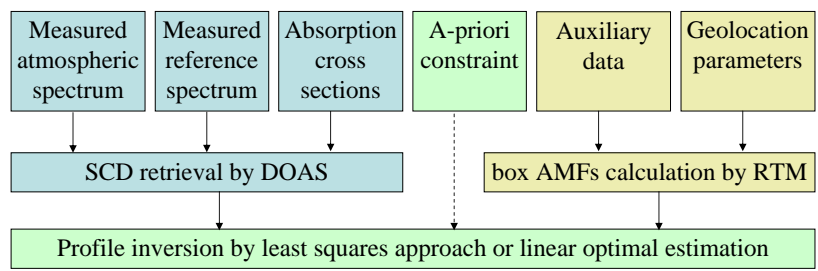

Fig. 1. Schematic diagram of the algorithm applied for profile retrieval.

The limb geometry is characterized by a very slant and thus long line of sight (LOS) through the atmosphere crossing extended volumes of air masses. In existing 1-D limb retrieval algorithms from SCIAMACHY limb spectra (Sioris et al., 2004; Rozanov et al., 2005; Kühl, 2005; Sinnhuber et al., 2005; von Savigny et al., 2005; Sioris et al., 2006; Pukīte et al., 2006; Kühl et al., 2007) the retrieval is performed for every limb scanning sequence separately, i.e. each corresponding profile is retrieved independently from the measurements of the previous or following scanning sequences.

However, large variability in the concentrations of photochemically active trace gases can occur along the long LOS: If the model assumes a horizontally homogeneous distribution, horizontal inhomogenity in the spatial distributions of atmospheric trace gases is introducing systematical errors in profile retrievals from limb measurements. Approaches to correct for this effect applying photochemical modelling have been described recently (Natarajan et al., 2005; McLinden et al., 2006; Sioris et al., 2006).

The aim of this study is to demonstrate a possibility to correct for the horizontal gradient effect from the observations themselves, applying a tomographic approach by combining consecutive limb scanning sequences that have a spatial overlap of their sensitivity regions. For infrared spectra, a direct inversion algorithm developed for the MIPAS instrument (also flown on ENVISAT) was introduced to retrieve temperature, pressure and trace gases simultaneously for all limb scanning sequences of one orbit taking into account the horizontal variability (Carlotti et al., 2001; Ridolfi et al., 2004; Carlotti et al., 2006). Also the infrared channels of OSIRIS are specifically designed for tomographic retrievals, although they are mainly used for measurements of terrestrial airglow emissions and not for limb-scattered radiation (Degenstein et al. (2003) and references on airglow emission tomography therein).

In this study a distance of only $3.75^{\circ}$ between satellite positions (corresponding to approx. $415 \mathrm{~km}$ along the Earth's surface) of consecutive scanning states for the northern part of a SCIAMACHY orbit will be used to study the impact of horizontal inhomogenity for limb measurements of scattered light in the UV/VIS spectral range. For that purpose, an overlap between the largely extended sensitivity regions of consecutive scanning sequences is utilized, i.e. we are taking advantage of the fact that the LOS for one particular scanning sequence crosses atmospheric volumes already probed by previous measuring sequences - under a different geometry.

This 2-D retrieval approach is a modification of our two step algorithm (Kühl, 2005; Puķīte et al., 2006; Kühl et al., 2007) where Differential Optical Absorption Spectroscopy (DOAS) and profile acquisition by applying radiative transfer modelling are performed in two separate steps. Similar approaches have been described by Haley et al. (2004), Krecl et al. (2006) and Sioris et al. (2006).

The 3-D fully spherical Monte Carlo radiative transfer modelling (RTM) allows the introduction of a 2-D box air mass factor concept, varying not only in altitude but also in latitude (for definition please see Sect. 4.3). This enables the estimation of sensitivity regions of limb measurements and allows the simultaneous inversion of many limb scanning sequences, thereby providing the 2-D field of spatial distribution of trace gas concentrations. In this article we investigate how the 2-D air mass factor concept can be applied to correct for the horizontal gradient effect.

The retrieved profiles which result from applying either 1-D or 2-D air mass factors (AMFs) are intercompared for selected case studies. The improvement is shown for both, selected cases of SCIAMACHY measurements as well as for model simulations.

\section{Retrieval algorithm}

An algorithm for the retrieval of $\mathrm{NO}_{2}, \mathrm{BrO}$ and $\mathrm{OClO}$ vertical profiles from SCIAMACHY limb measurements was developed in our group (Kühl, 2005; Puķīte et al., 2006; Kühl et al., 2007). It allows the efficient retrieval of trace gas profiles, and shows a good agreement of the retrieved $\mathrm{BrO}$ and $\mathrm{NO}_{2}$ profiles with balloon measurements (Dorf et al., 2006; Butz et al., 2006; Kühl et al., 2007). The retrieval of vertical trace gas profiles from SCIAMACHY measured limb spectra is done in two steps as illustrated in Fig. 1. In the first step, slant column densities (SCDs), the integrated concentration of the absorber along the light path, are derived from the SCIAMACHY limb spectra by DOAS. For OCIO the fitwindow ranges from 363.5 to $391 \mathrm{~nm}$ and for $\mathrm{NO}_{2}$ from 420 to $450 \mathrm{~nm}$. As reference spectrum we use a measurement at a tangent height where the absorption of the considered trace gas is small $\left(\sim 36 \mathrm{~km}\right.$ for $\mathrm{OClO}$ and $\sim 42 \mathrm{~km}$ for $\left.\mathrm{NO}_{2}\right)$. The small abundances of the considered absorbers which appear at the tangent height of the reference spectrum are estimated by a latitude dependent a-priori and their impact is added to the retrieved SCDs.

Second, the trace gas SCDs are converted into vertical concentration profiles applying RTM. To increase the signalto-noise ratio only one averaged SCD per tangent height is applied for the inversion, which is performed either by the 
optimal estimation method (Rodgers, 2000) or a least squares approach (Menke, 1999).

For the algorithm details please refer to Puksite et al. (2006) or more recently to Kühl et al. (2007). Being the most relevant for the issues of this article, specific details of the RTM are given in the next two sections and also in the Appendix.

\section{Radiative transfer modelling}

\subsection{Spatial sensitivity}

The instrument measures light scattered into the LOS either directly from the incoming solar radiation, or being scattered previously by the atmosphere, clouds or the ground below. The limb geometry is characterized by relatively long paths of light along the LOS after the last scattering event, in comparison to the paths before the atmospheric last scattering event when solar zenith angle (SZA) is smaller than $90^{\circ}$. Along the LOS the instrument has different sensitivities for different locations in the atmosphere. In general, the instrument exhibits a higher sensitivity to air masses closer to the instrument since the light contributing to the measurement integrates along the LOS (see schematic view in Fig. 2). On average, one will get gradually increasing sensitivity for the LOS towards the instrument.

Another factor is that the asymmetry of the sensitivity region increases for rising optical depths and therefore with decreasing tangent height (mainly due to scattering on air molecules). Also absorption (especially ozone) and scattering by aerosols, clouds and reflection at the ground modify the measured light intensity.

For high altitudes, where the atmosphere is optically transparent, a nearly symmetrical distribution across the TP of photons being scattered into the LOS is observed by the model: Nearly one half of all photons contributing to the measurement are scattered into LOS between TP and instrument (near limb side), the other half from behind the TP (far limb side).

For the retrieval at low altitudes a limiting factor is the large probability for Rayleigh scattering i.e. the atmosphere is optically thick. Furthermore, usually clouds are present along the LOS at low altitudes, also preventing sensitivity for low atmospheric layers.

Therefore at low tangent heights with an optically dense atmosphere, more photons contribute from volumes of the side between TP and instrument. Thus, besides the low sensitivity for altitudes below $12-15 \mathrm{~km}$, a larger shift of the sensitivity towards the near limb side occurs. This also means (as it will be seen later in Sect. 4.3) that the measured spectra practically contain no information about regions around the TP for low tangent heights.

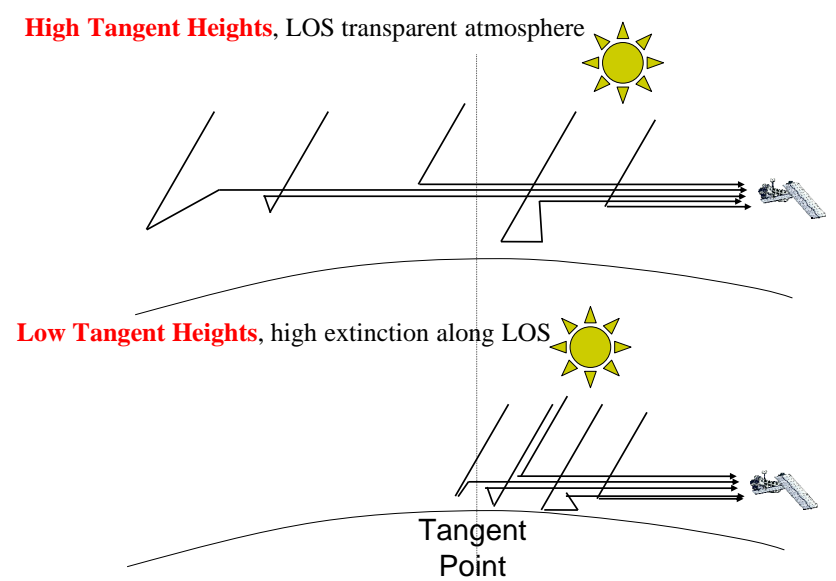

Fig. 2. Schematic view of the spatial sensitivity distribution. Contributing light paths are displayed. More crossing paths means higher sensitivity for a particular region (note that the impact of the considered absorber on the detected slant column density increases according to the number of light paths).

\subsection{Box air mass factors}

To retrieve the desired trace gas profile from the SCDs derived by DOAS, the relation between these two quantities needs to be established. The box AMFs quantify the spatial sensitivity for certain space regions (or boxes) in the atmosphere and relate the measurement space (SCDs) to the model space (vertical column densities (VCDs), the number density multiplied by the vertical extension of the box). In matrix form the relation is:

$$
S C D=A M F \times V C D
$$

where the elements of $\boldsymbol{S C D}$ are SCDs belonging to certain observation geometries (tangent heights, instrument positions and viewing angles), the elements of $\boldsymbol{V} \boldsymbol{C} \boldsymbol{D}$ are VCDs belonging to certain boxes and the box AMFs matrix AMF quantifies the impact of the VCDs of these boxes on the SCDs.

In terms of the Lambert-Beer Law the SCD of an absorber of interest at a certain viewing geometry $g$ can be expressed as:

$S C D_{g}=-\frac{\log \left(\frac{I_{g}}{I_{g 0}}\right)}{\sigma}$

where $\sigma$ is the absorption cross-section of the tracegas, $I_{g}$ is the intensity observed for the geometry $g$ and $I_{g 0}$ is the intensity without the absorber of interest.

For a certain box $b$ the box AMF $A M F_{g b}$ describes the impact of the $V C D_{b}$ within that box on the measured $S C D_{g}$ at the geometry $g$. Hence, in order to assess this impact one needs to calculate derivatives of SCDs with respect to the 
$V C D_{b}$. In practice this means to calculate the derivative of the logarithm of the intensity with respect to the number density $n_{b}$ in the box $b$ or, more precisely, the absorption coefficient $\beta_{b}$ :

$A M F_{g b}=\frac{d S C D_{g}}{d V C D_{b}}=-\frac{1}{h_{b} \sigma_{b}} \frac{d \log I_{g}}{d n_{b}}=-\frac{1}{h_{b}} \frac{d \log I_{g}}{d \beta_{b}}$

where $\sigma_{b}$ is the absorption cross-section of the considered trace gas in the box $b$. In this study box AMFs are calculated as derivative of the logarithm of the intensity with respect to the absorption coefficient (last term in Eq. (3)), normalized by the vertical extension $h_{b}$ of the box.

\subsection{Radiative transfer model "Tracy-II"}

We apply the 3-D fully spherical Monte Carlo RTM "TracyII" (Deutschmann and Wagner, 2007; Wagner et al., 2007) to calculate box AMFs. The largest advantage of Monte Carlo models in limb geometry is that they properly take into account Earth's sphericity both for single and multiple scattering (Oikarinen et al., 1999; Loughman et al., 2004). Furthermore they also provide the possibility to simulate an inhomogeneous atmosphere up to a high degree. The model does not take into account refraction since it would require additional computer power but its effect was found to be negligible for altitudes above $12 \mathrm{~km}$ (see e.g. Sioris et al., 2006). Also aerosols and clouds are not included in this study. The aerosol extinction is much lower compared to extinction by Rayleigh scattering in the stratosphere. Also, due to the slantness of limb observations, SCDs derived from measured spectra are practically insensitive to the atmosphere below the tangent height.

For temperature, pressure and ozone we apply a model simulation provided by Brühl and Crutzen (1993). It should be noted that in some individual cases the actual temperature, pressure and ozone profile might differ considerably from the assumed model profiles. From sensitivity studies we found that the related errors in profile retrieval can be up to $10 \%$. However, the conclusions of this study are not affected by these systematic effects.

The whole process of RTM in "Tracy-II" is separated into two parts: backward trajectory generation and weighting. The multiple scattering is performed until the modelled light trajectories leave the atmosphere. The scattering angle is selected according to the phase function of the respective scattering event using random numbers. For the path generation scheme the interested reader is referred to Marchuk et al. (1980) or Davis and Knyazikhin (2005). The peculiarity of our algorithm is to separate scattering and absorption processes. The trajectory generation does not produce any absorption event, therefore the absorption effect is determined in the second step (weighting) for the simulated photon paths. The advantage of this method is, that one photon ensemble can be used for an arbitrary absorption scenario.
A more detailed description on the weighting of generated trajectories and its relation to the calculation of box AMFs is provided in the Appendix.

\section{Two-dimensional retrieval}

\subsection{Horizontal gradients}

Photochemically active species like $\mathrm{BrO}, \mathrm{NO}_{2}$ and $\mathrm{OClO}$ can vary significantly in space and time due to their dependence on solar illumination, atmospheric chemistry and transport. The large volume to which satellite limb observations are sensitive requires the consideration of gradients in the trace gas distributions. Since the instrument is more sensitive to the air masses closer to it, the near limb side will have a larger effect on the measurement results. If horizontal gradients exist, algorithms which do not account for the horizontal variation of trace gases will introduce errors in the retrieval: If there are higher concentration values towards the instrument (in comparison to the TP) these will be wrongly accounted for the location where the measurement is assumed to be taken (for the TP). Therefore a positive horizontal gradient will - in the 1-D retrieval - lead to a concentration higher than in reality and the peak values will tend to appear at lower altitude: For negative gradients the opposite is the case.

Algorithms which assume homogeneous horizontal distributions do not take into account that the LOS and hence the light, which contributes to measurement before and after being scattered into the LOS, crosses regions with concentrations different to those appearing around TP. For situations with significant horizontally inhomogeneous distribution this will lead to increased systematic errors in the profile retrieval.

\subsection{The retrieval approach}

In order to account for possible gradients of the considered trace gas along the flight/viewing direction, we propose a two-dimensional tomography-based retrieval approach: The individual measurements are described as a superposition not only regarding varying altitude (as in 1-D retrievals) but also regarding latitude parameters. I.e. SCDs derived from consecutive measurement sequences of one orbit are inverted simultaneously, including in the retrieval the information about the horizontal sensitivity of the measurements.

With this approach a better description of the impact of the viewing geometry on the measurement is realized: The abundance of the considered trace gas measured along the LOS is not assumed to occur at the TP only (as in the 1-D retrieval) but distributed along the LOS taking into account the sensitivity of the instrument. Thus, the 2-D approach distinguishes between air volumes at different regions along LOS. Therefore the retrieved profiles will agree better with reality (unless there are errors in the algorithm). 

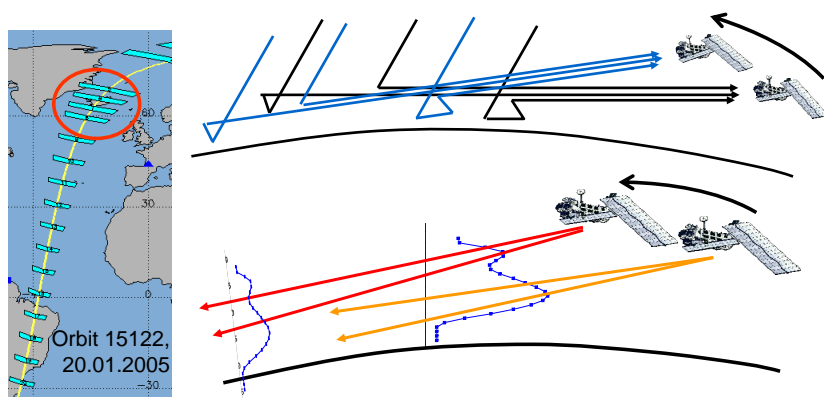

Fig. 3. Spatial correlation of two successive limb scanning sequences (right panel) together with a geolocation map of the SCIAMACHY limb scanning sequences (left panel, taken from SOST webpage: atmos.caf.dlr.de/projects/scops/). If the distance is small enough for the measurement regions to partially overlap, as for scanning sequences indicated with red circle (left panel), the measurement of the same air masses is made from different instrument positions.

A precondition for an improvement with respect to the 1-D algorithm is that the change of the atmospheric trace gas concentrations (particularly because of SZA change and transport of air) in time is negligible. The SZA change in time (in January) for consecutive scanning sequence in the North is from $\sim 0.025^{\circ}$ (most northern state) to $\sim 0.07^{\circ}$ (around $\left.60^{\circ} \mathrm{N}\right)$ during the time $(\sim 1 \mathrm{~min})$ which is necessary to cross the distance between two consecutive scanning sequences (Fig. 3, left panel indicated with red circle). This difference in the SZA does not result in significant profile changes of the considered absorbers.

Also the spatial distance between the consecutive scanning sequences should be small enough so that they overlap in some extent (see Fig. 3) and be at least less than the sensitivity region of one limb measurement. Both criteria are fulfilled for the northern part of SCIAMACHY orbits, where the first 3 or 4 limb scanning sequences (indicated for an example in the right panel of Fig. 3) are performed without nadir observations in between.

The SZA change per minute during a SCIAMACHY orbit increases until the equator is reached, with its maximum of $\sim 0.25^{\circ}$ (in January) and then decreases again southwards.

\subsection{Two-dimensional box air mass factors}

The light path lengths in a box depend on the form of the box only. Hence Eq. (3) is valid for any dimension of the box $b$, it can be either 1-D, 2-D or even 3-D.

Figure 4 shows 1-D box AMFs calculated by the RTM Tracy-II as a function of the tangent height. The box AMFs depicted in Fig. 4 demonstrate only the sensitivity to vertically resolved but horizontally homogeneous atmospheric layers. In this case, the measured SCD at a certain geometry

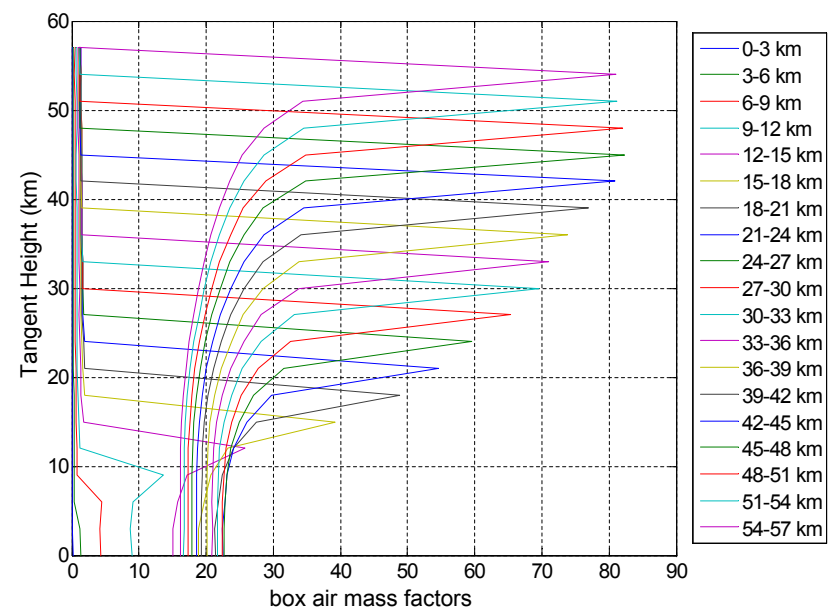

Fig. 4. One dimensional box AMFs for $435 \mathrm{~nm}$ plotted for $3 \mathrm{~km}$ thick boxes as function of the tangent height. The illustration is for the 3 rd scanning sequence (solar zenith angle: $84^{\circ}$; solar azimuth angle: $-43^{\circ}$ at the tangent point) in the descending part from North of an orbit of SCIAMACHY in the middle of January (orbit 15122 on 20 January 2005). The peak value usually is located at the tangent height equal to the altitude of the "box" simulated.

$g$ is defined as:

$S C D_{g}=h \sum_{b_{a l t}} A M F_{g, b_{a l t}} n_{a l t}$

where $A M F_{g, b_{a l t}}$ and $n_{\text {alt }}$ are box AMFs resolved in altitude alt for the geometry $g$ and number density, respectively. $h$ is the vertical extension used for the boxes.

In our 2-D retrieval the box AMFs vary not only in altitude but also in latitude. Here the $S C D_{g}$ is described as a sum of products of both, in altitude and latitude lat resolved box AMFs and number densities:

$S C D_{g}=h \sum_{b_{\text {alt }, l a t}} A M F_{g, b_{\text {alt }, l a t}} n_{\text {alt }, \text { lat }}$

Due to the additional latitudinal dimension, the box AMFs calculation time of "Tracy-II" increases by a factor of 1.5.

The 2-D box AMFs describe the spatial character of the sensitivity of limb measurements in a more appropriate way, see Fig. 5. The enhanced sensitivity for regions crossed by the LOS can be nicely seen; the higher sensitivity for the instrument side can be realized, too. The spatial distribution of 2-D box AMFs for different tangent heights of the instrument's LOS is depicted in Fig. 6 for a pure Rayleigh atmosphere, i.e. without clouds and aerosols. The latitudinal borders of the boxes are selected as midpoints between the tangent points (TP) of the scanning sequences of the instrument.

Due to increased Rayleigh scattering at low tangent heights of the LOS (see the example given for a tangent 
$1 \mathrm{dim}$

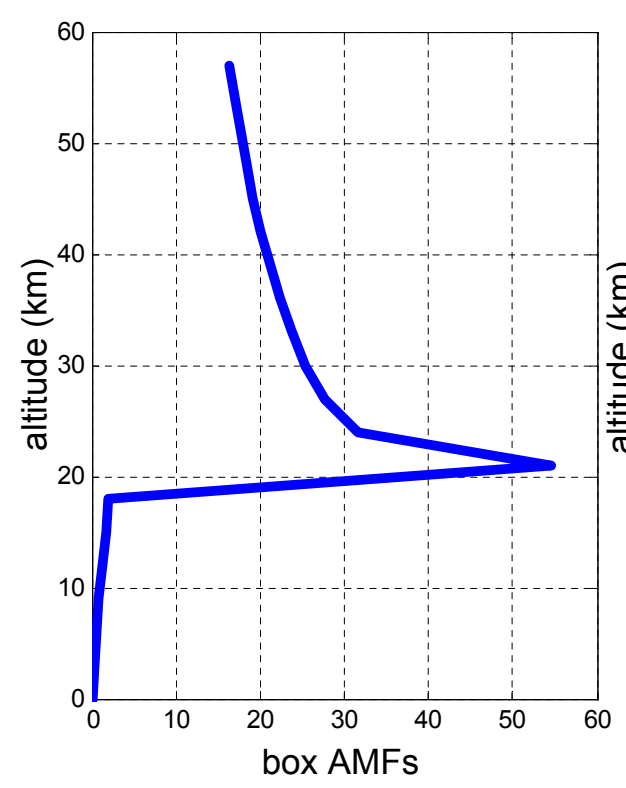

$2 \operatorname{dim}$

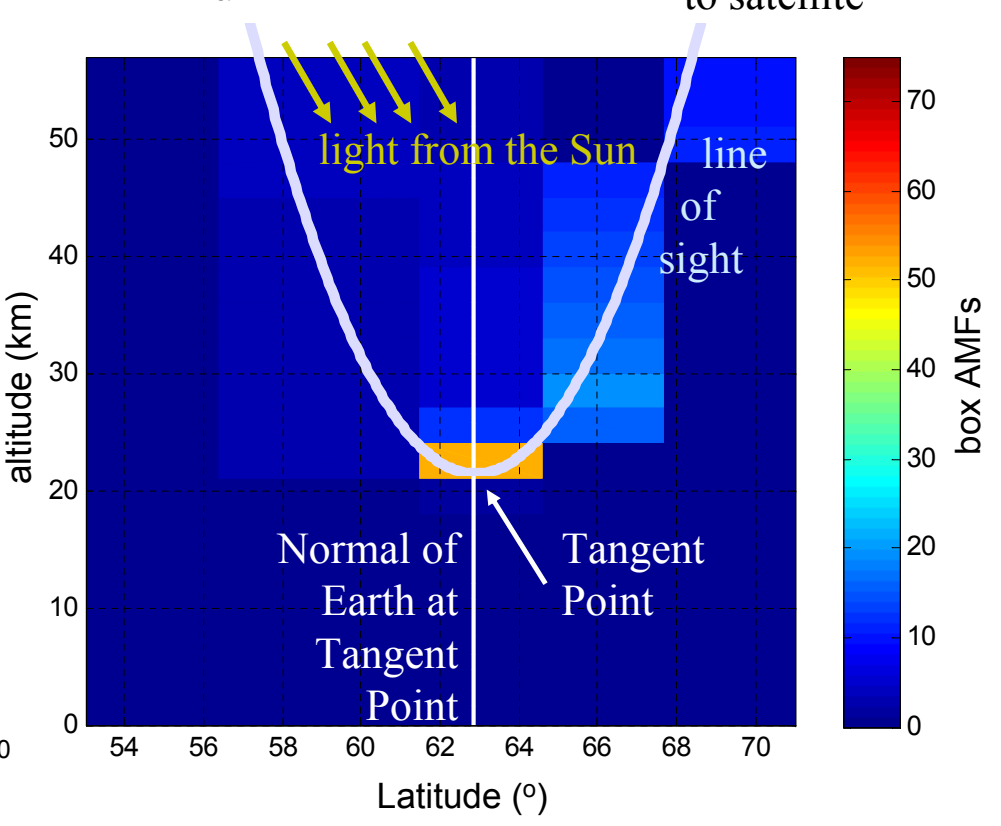

Fig. 5. Comparison between 1-D and 2-D box air mass factors. Values calculated for $435 \mathrm{~nm}$ are plotted for tangent height at $21 \mathrm{~km}$. Illustrations are for the 3rd scanning sequence (solar zenith angle: $84^{\circ}$; solar azimuth angle: $-43^{\circ}$ at the tangent point) in the descending part from North of an orbit of SCIAMACHY in the middle of January (orbit 15122 on 20 January 2005). The latitudinal distribution of the air mass factor along the line of sight of the instrument can be realized for 2-D box AMFs. Introducing 2-D AMFs in the retrieval is distinguishing between air masses measured not only in altitude but also in latitude. $1^{\circ}$ of latitude corresponds to $\sim 119-160 \mathrm{~km}$ depending on line of sight azimuth angle. Line of sight, normal of the Earth, tangent point, and direction of Sun light are also shown in the figure.

height of $9 \mathrm{~km}$ in the Fig. 6) the contribution of light to the measurement is small for areas close to the TP (for the given example the TP is at $\left.\sim 63^{\circ} \mathrm{N}\right)$. Therefore the sensitivity for low altitudes $(<15 \mathrm{~km})$ around the TP is low. The sensitivity increases with tangent height. Since the box AMFs depend on the slantness of the light paths, large values and thus high sensitivity for high tangent heights is found around the TP. The fact that the light after the last scattering event propagates towards the instrument along a very slant trajectory leads to high values of 2-D box AMFs along the LOS.

Figure 6 illustrates also the wavelength dependency of the box AMFs: Due to a larger probability of Rayleigh scattering for smaller wavelengths the box AMFs at $380 \mathrm{~nm}$ are decreased with respect to the box AMFs at $435 \mathrm{~nm}$.

\section{Results}

As an example we show retrievals of $\mathrm{NO}_{2}$ and $\mathrm{OClO}$ number density profiles for selected orbits in January 2005. The retrieval is performed applying either 1-D or 2-D box AMFs in order to investigate if the 2-D box AMF concept is changing the profile retrieval to the direction expected by the considerations in Sect. 4.1: This is awaited because of the improved description of the reality in the forward model for situations where the distribution of the considered trace gas along the LOS is inhomogeneous. The left panel in Fig. 7 shows the geolocation of the tangent points corresponding to the limb measurement sequences of orbit 15122 on 20 January 2005. The right panel gives an illustration of the potential vorticity at the $475 \mathrm{~K}$ level (approx. $19 \mathrm{~km}$ altitude) above the North Pole for the same day. The position of the considered SCIAMACHY observations in the northern part of the selected orbit is indicated with a red square in both maps (approx. $60^{\circ}$ to $\left.70^{\circ} \mathrm{N}\right)$.

The interesting limb scanning sequences are located at the boundary of the polar vortex. Here, due to denoxification inside the polar vortex, a strong negative gradient along the LOS (from the TP towards instrument) is expected for $\mathrm{NO}_{2}$. Vice versa, for $\mathrm{OClO}$ a positive gradient is expected due to strong chlorine activation inside the polar vortex in the extraordinary cold January 2005 (regarding stratospheric temperatures). The retrieved $\mathrm{NO}_{2}$ and $\mathrm{OClO}$ number densities for the northern part of the selected orbit are plotted as a function of latitude and altitude, both for 1-D and 2-D approach, in Figs. 8 and 9 (left and right panels, respectively). It can be seen very clearly that the $\mathrm{NO}_{2}$ number density is decreasing monotonically from $60^{\circ}$ to $70^{\circ} \mathrm{N}$ (in particular at the peak altitude of approx. $28 \mathrm{~km}$ ). For $\mathrm{OClO}$, a rapid 

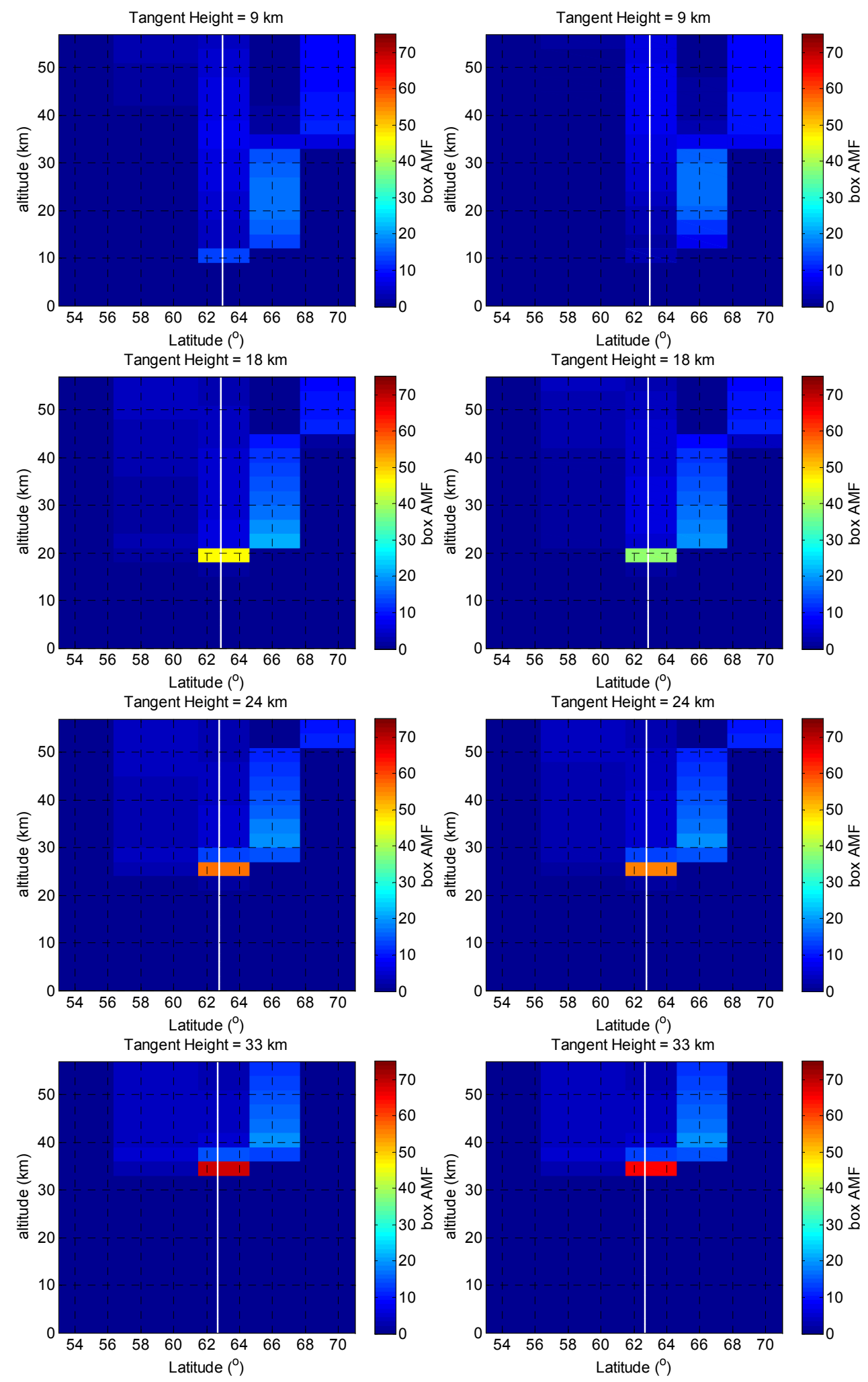

Fig. 6. 2-D AMFs for different tangent heights as modelled by the RTM model Tracy-II for $435 \mathrm{~nm}\left(\mathrm{NO}_{2}\right)$ on the left panel and $380 \mathrm{~nm}$ $(\mathrm{OClO})$ on the right panel. Values are plotted for the example of the 3rd scanning sequence in the descending part from North of an orbit of SCIAMACHY in the middle of January (orbit 15122 on 20 January 2005). The normal from tangent point to the Earth surface is indicated with a white line. 


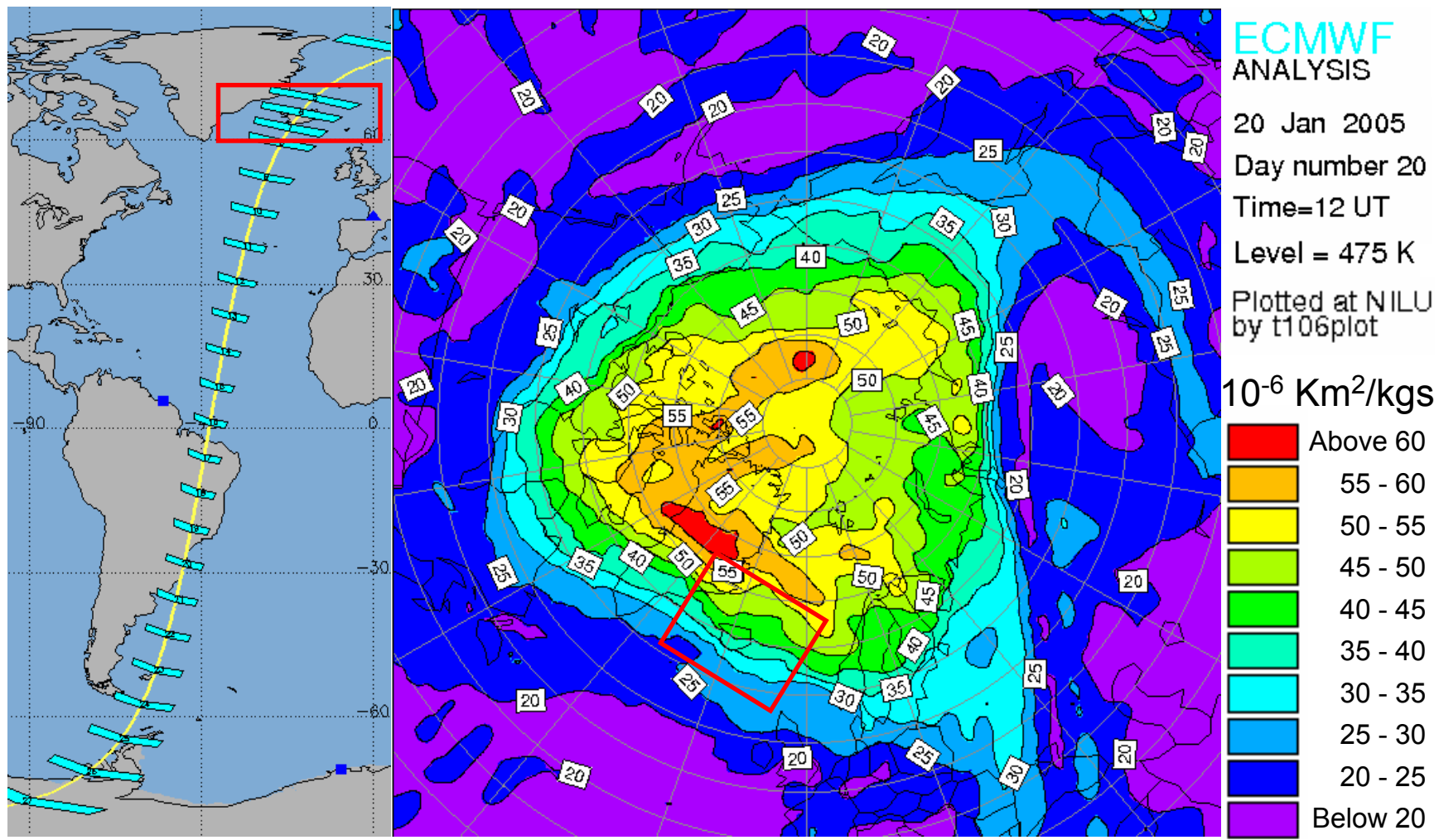

Fig. 7. Left panel shows geolocations of the tangent points for the limb scanning sequences of the orbit 15122 on 20 January 2005 . The red box indicates the states for which the retrieval is shown in the line plots in Fig. 8. Potential vorticity for the same day, 12:00 UT is displayed in the right panel (ECMWF, 2000).

increase is observed from $65^{\circ}$ to $70^{\circ} \mathrm{N}$ for altitudes between 15 and $18 \mathrm{~km}$. Comparing the retrieved profiles for the 1 $\mathrm{D}$ and 2-D approach (dashed lines in Fig. 8 and lower panel in Fig. 9), significant differences appear. For the $\mathrm{NO}_{2}$ retrieval (left panels of Figs. 8 and 9) an increase of the values in the 2-D approach is visible for altitudes from 21 to $30 \mathrm{~km}$ and latitude regions of $61.5-64.5^{\circ}$ and $64.5-67.5^{\circ}$ (dashed red and green lines, respectively). The typical difference between both retrievals is about $20 \%$.

As can be seen from Fig. 6, the LOS for the 3rd scanning sequence from North (as indicated by the red box in the left panel of Fig. 7 with TP located at $\sim 63^{\circ} \mathrm{N}$ ) crosses also regions of $64.5-67.5^{\circ} \mathrm{N}$ for a wide altitude range $(\sim 20 \mathrm{~km})$. In the 1-D retrieval, where a homogeneous distribution is assumed, the SCD obtained in the first step of the retrieval (see Sect. 2) is interpreted only as a superposition of the trace gas abundances at different altitude levels. Therefore, also horizontal gradients will be wrongly accounted as vertical number density changes. In other words, a decreased measurement value of SCD due to lower $\mathrm{NO}_{2}$ values towards the instrument will incorrectly be interpreted as a decrease in concentration values retrieved and assumed for the TP. However in the 2-D retrieval approach, the model uses knowledge from the previous state assigning a part of the measured SCDs to lower concentrations more to the North.
For the same example of consecutive limb scanning sequences in the northern part of the orbit 15122 we applied the 2-D approach on the retrieval of $\mathrm{OClO}$ profiles (see right panels in the Figs. 8 and 9). Although the relative retrieval errors for $\mathrm{OClO}$ are larger (in comparison with $\mathrm{NO}_{2}$ retrieval), systematically lower concentrations for the 2-D retrieval of $\mathrm{OClO}$ can be observed for latitudes below $\sim 67.5^{\circ} \mathrm{N}$ (or for regions $64.5-67.5^{\circ} \mathrm{N}$ (green line) and $61.5-64.5^{\circ} \mathrm{N}$ (red line) corresponding to the second and third scanning sequence). Hence the 2-D approach results in a more rapid decrease of $\mathrm{OClO}$ when leaving polar vortex, which is in better agreement with the expectations. Similarly, the strongest gradient of $\mathrm{NO}_{2}$ in orbit 15122 (see Figs. 8 and 9) occurs when crossing the border of Arctic polar vortex (compare Fig. 7, right panel). Also the rapid decrease of $\mathrm{OClO}$ is observed at the location of this barrier for horizontal transport (see Figs. 8 and 9). Other examples where strong gradients of $\mathrm{NO}_{2}$ occur, are depicted in Fig. 10 for orbits 14979 on 10 January 2005 (panel a), 15080 on 17 January 2005 (panel b), 15088 on 18 January 2005 (panel c) and 15149 on 22 January 2005 (panel d).

The largest differences between the 1-D and the 2-D approaches occur if the gradient is strong and appears for an extended altitude interval, see e.g. results for orbit 14979 (latitude regions corresponding to scanning sequences 3 and 

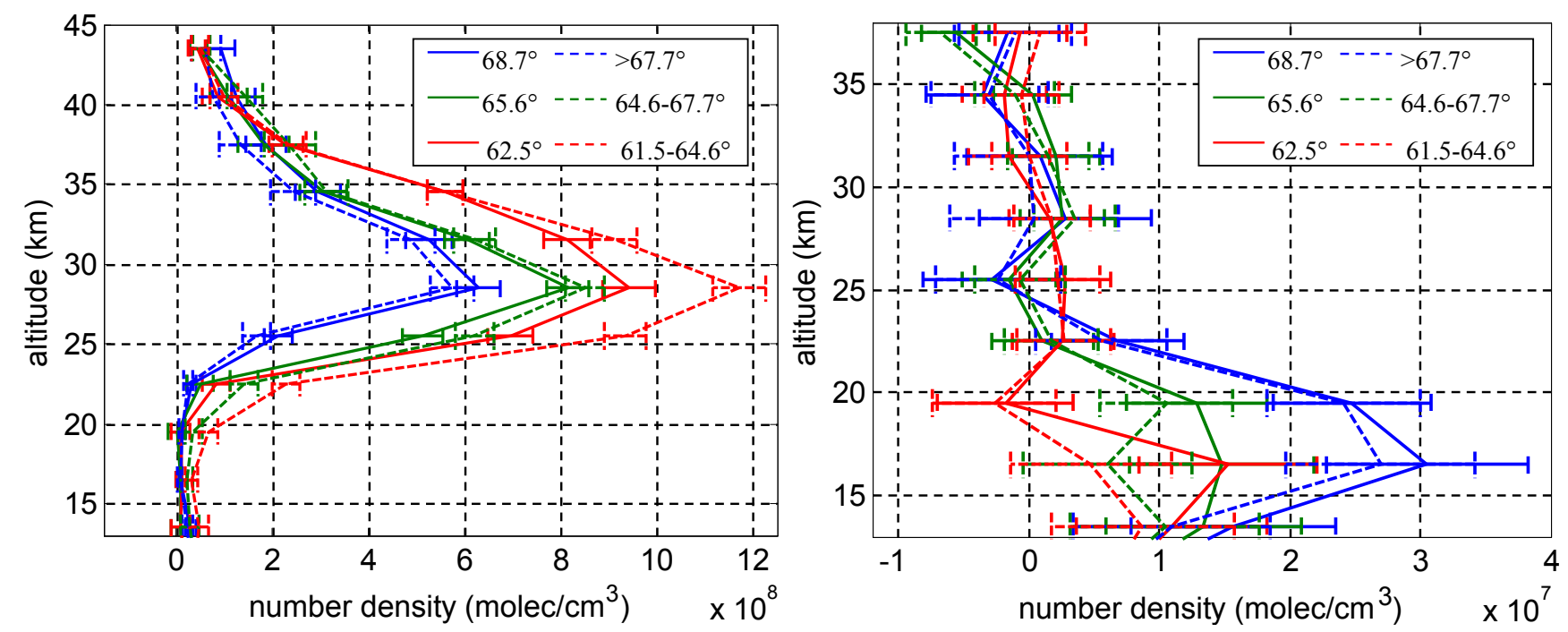

Fig. 8. Comparison between retrieved profiles using 1-D approach (solid lines) and 2-D approach (dashed lines) for orbit 15122 on 20 January 2005 for $\mathrm{NO}_{2}$ (left panel) and OClO (right panel) for the first three northern states of descending part of the orbit (see also Figs. 7 and 9).
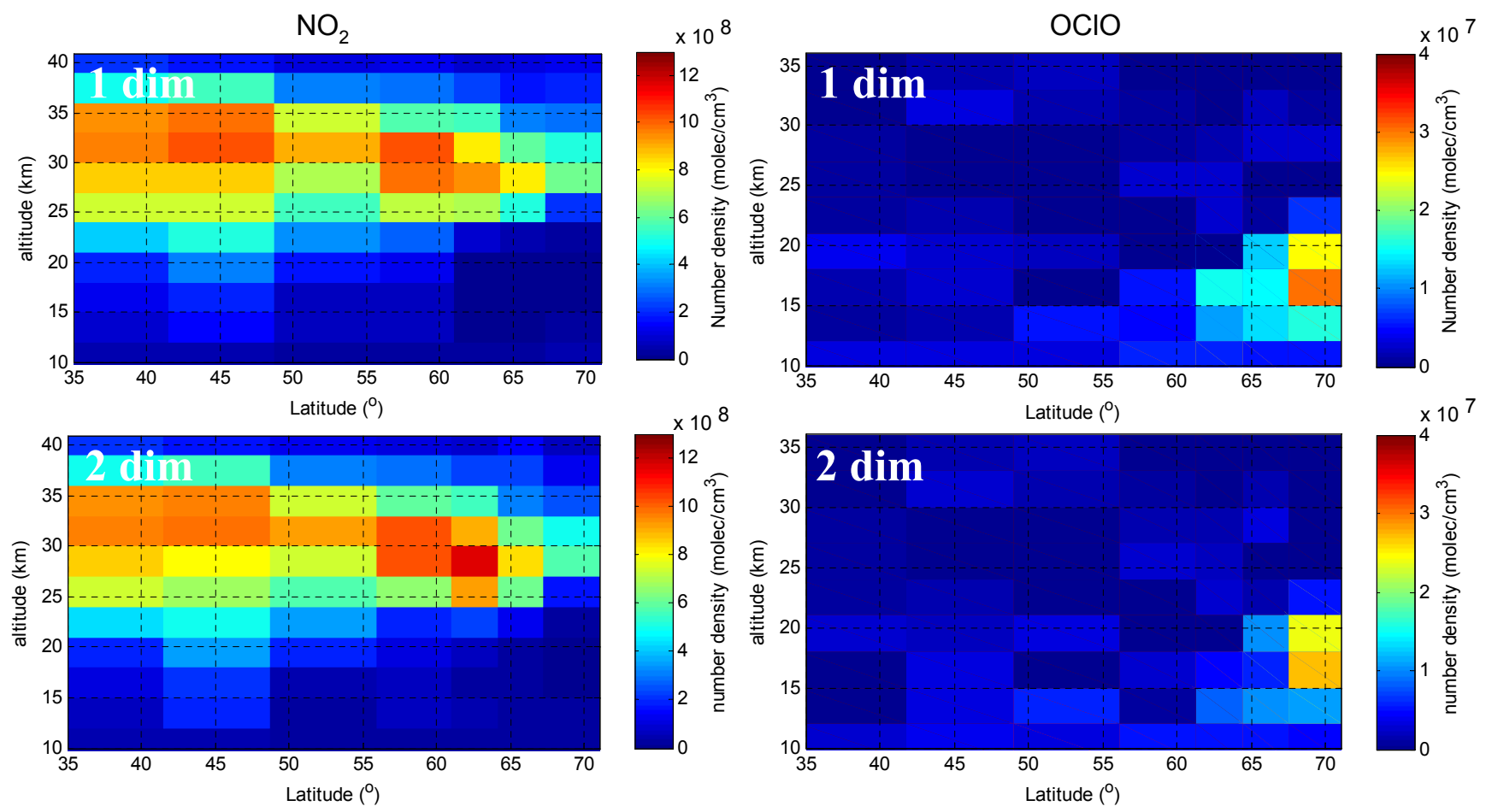

Fig. 9. Comparison between retrieved profiles using 1-D approach (upper panel) and 2-D approach (bottom line) for orbit 15122 on 20 January 2005 for $\mathrm{NO}_{2}$ (left panel) and OClO (right panel) for northern part of orbit 15122 (see also Figs. 7 and 8). For the 2-D retrieval with respect to the 1-D approach larger horizontal gradients are achieved, in agreement to the expectations for the vortex edge. In addition, for the most northern scanning sequences also a higher latitudinal resolution can be obtained. 

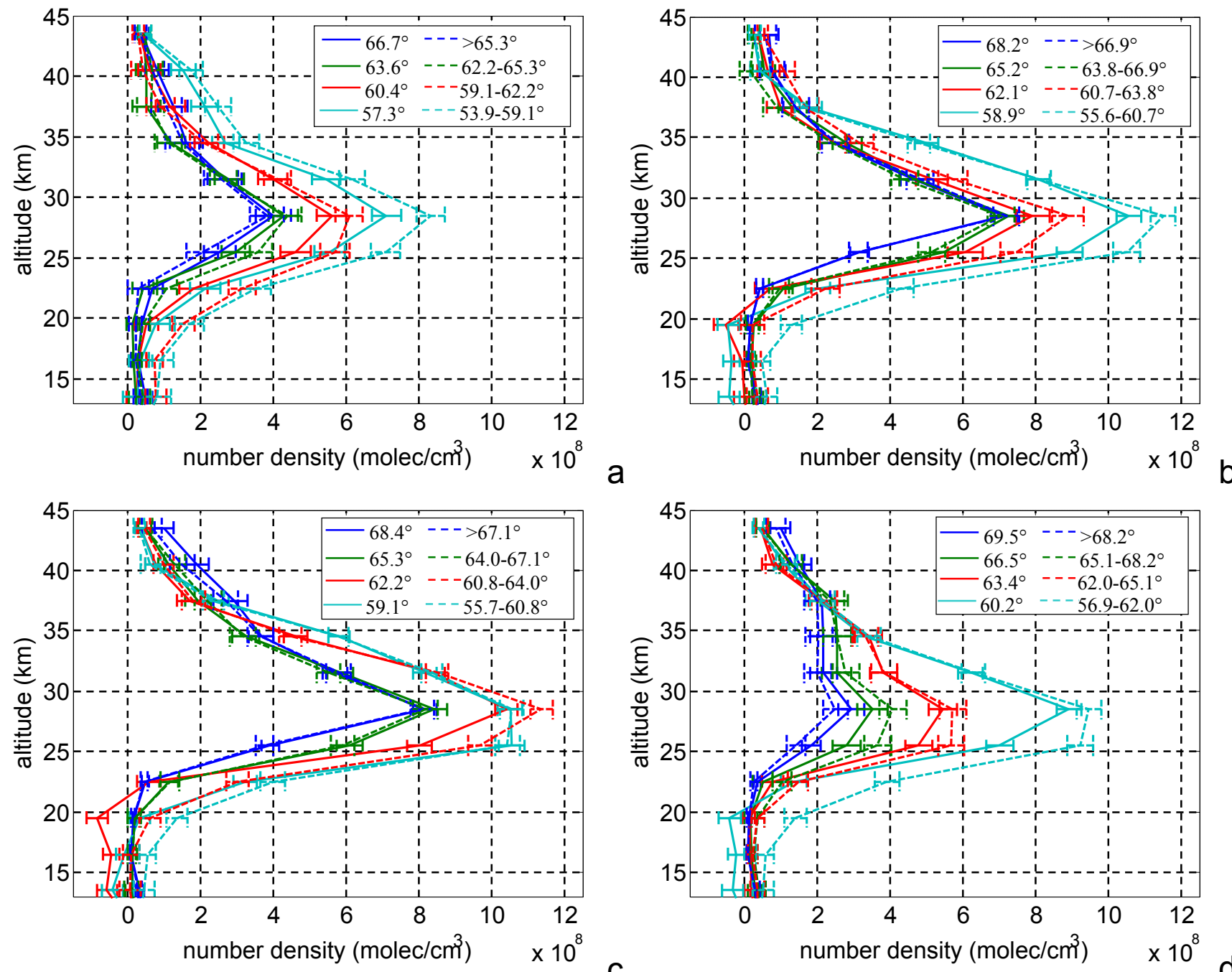

Fig. 10. Comparison between retrieved profiles of $\mathrm{NO}_{2}$ made separately for every scanning sequence i.e. applying 1-D box air mass factors (solid lines) or performing profile inversions for all scanning sequences at the same time i.e. applying 2-D box air mass factors (dashed lines). Results for the first 4 latitudinal regions (2-D retrieval) or scanning sequences (1-D retrieval) are displayed as they follow from the North in blue, green, red and cyan. The results are displayed for orbits 14979 on 10 January 2005 (panel a), 15080 on 17 January 2005 (panel b), 15088 on 18 January 2005 (panel c) and 15149 on 22 January 2005 (panel d ).

4), results for orbits 15080 and 15149 (latitude regions corresponding to scanning sequences 2 and 3 and also 3 and 4), and the results for orbit 15088 (scanning sequences 2 and 3), shown in Fig. 10. It can be seen that the disagreement between the 1-D and 2-D retrievals increases for lower altitudes if a strong gradient exists. Also for OClO large differences between 1-D and 2-D retrievals can be found as illustrated in Fig. 11. For scanning sequences crossing the polar vortex boundary a decrease in the $\mathrm{OClO}$ concentrations of approximately $50 \%$ is found.

\section{Verification}

In order to verify that the profiles derived by the 2-D approach are indeed resulting from an improved description of the measurement sensitivity regions, we also selected a case (orbit 15146 on 22 January 2005) where practically no gradient for $\mathrm{NO}_{2}$ is observed for the most northern part of orbit, see Fig. 12. Here the retrieval applying either 1-D or 2-D box AMFs results in very similar profiles indicating that the change in the cases above is not an artefact.

Furthermore, we also tested our 1-D and 2-D retrievals using modelled trace gas fields. For that purpose we chose simple scenarios in which the gradient (either negative or 

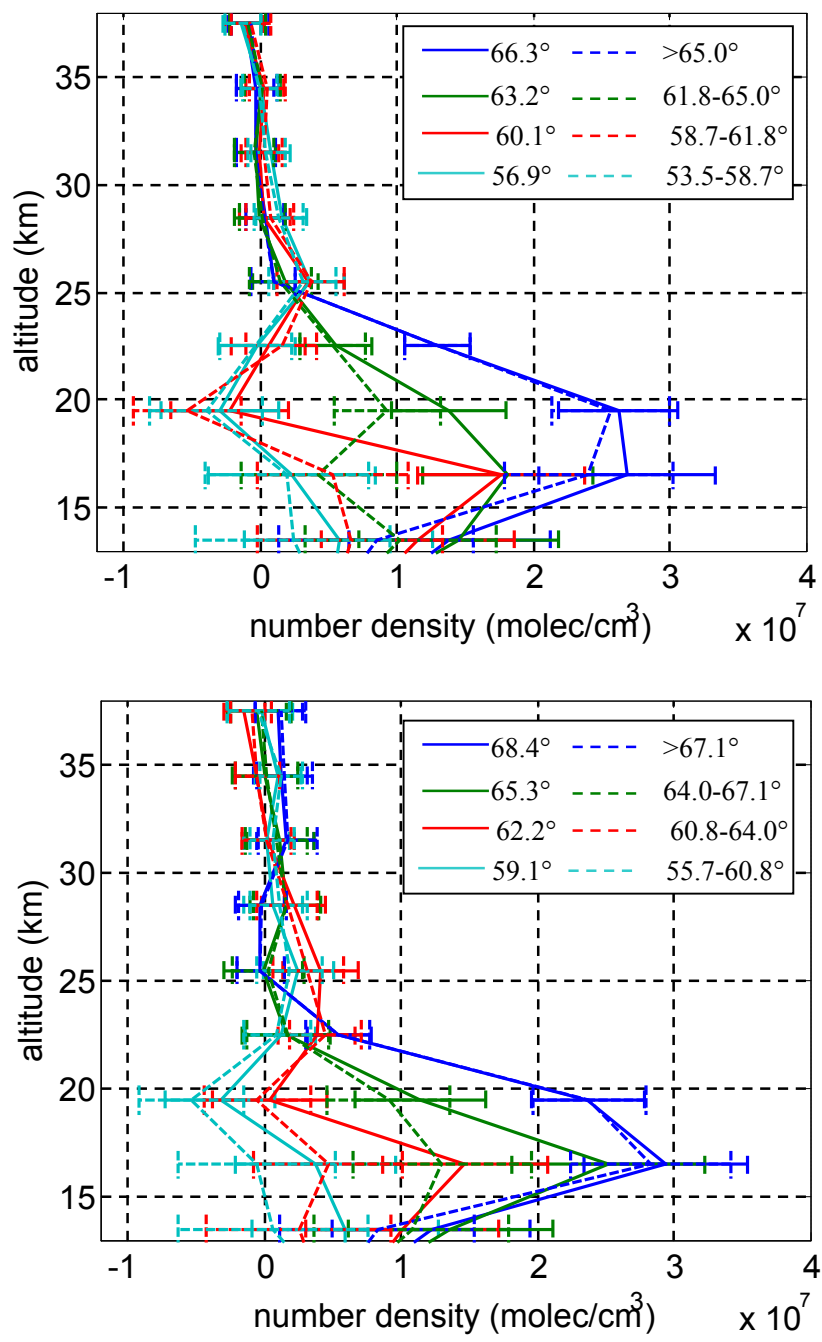
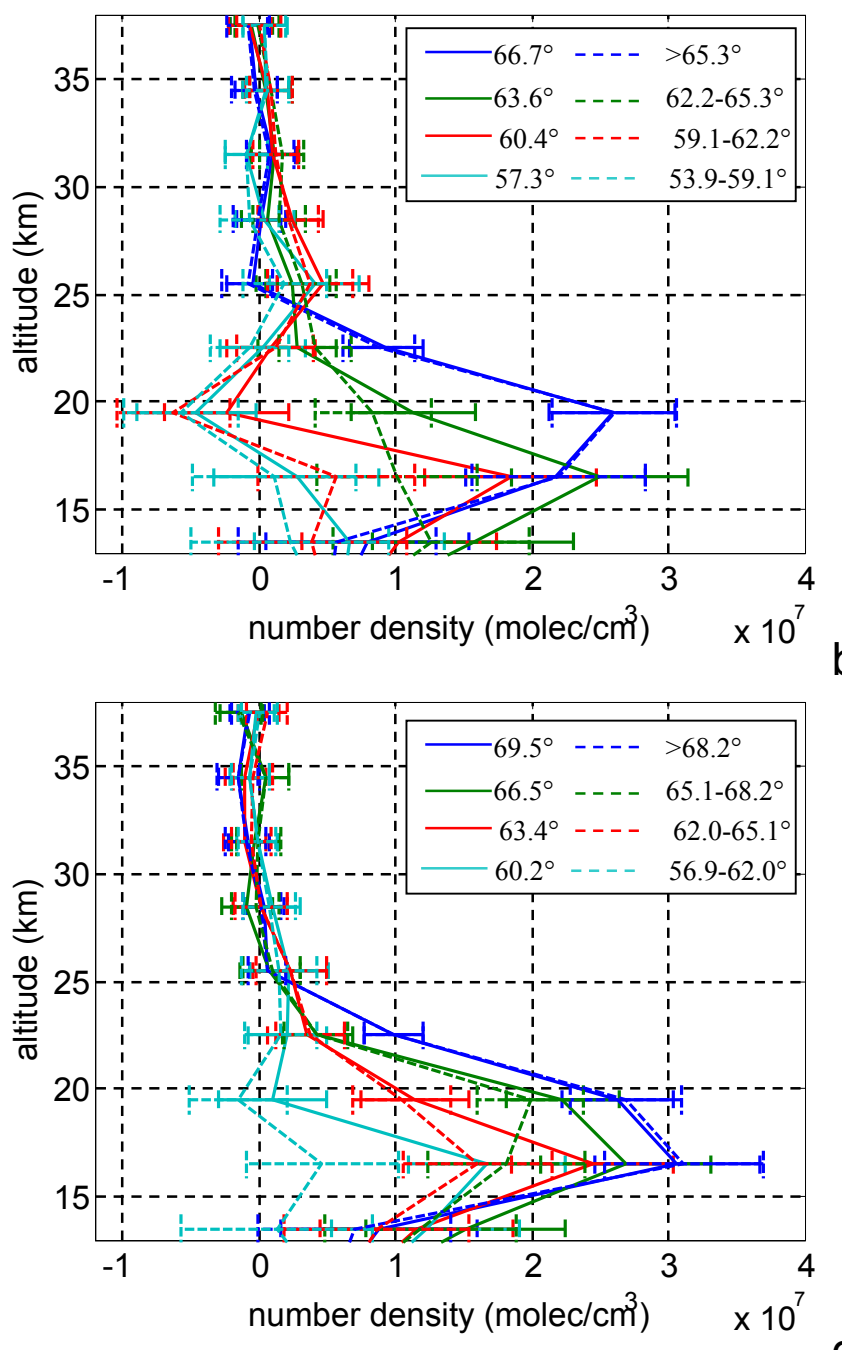

Fig. 11. The same as in Fig. 10 but for OClO for orbits 14951 on 8 January 2005 (panel a), 14979 on 10 January 2005 (panel b), 15088 on 18 January 2005 (panel c) and 15149 on 22 January 2005 (panel d).

positive towards the instrument) are prescribed by well known number density values. We then applied "Tracy-II" to simulate the corresponding SCDs for these stratospheric trace gas fields, adopting the geometry of SCIAMACHY orbit 15122 . For the first study (the negative gradient towards the instrument) we prescribed a trace gas distribution as depicted in Fig. 13 (upper panel, left). A stepwise gradient on a five times finer latitudinal grid than the measurement grid of SCIAMACHY was selected and a Gaussian shape profile with peak at $28.5 \mathrm{~km}$ and $\sigma$-parameter of $4 \mathrm{~km}$ (FWHM $\sim 9.4 \mathrm{~km}$ ) was assumed. The gradient was from $3 \times 10^{8} \mathrm{molec} / \mathrm{cm}^{3}$ at $71^{\circ} \mathrm{N}$ to $12 \times 10^{8} \mathrm{molec} / \mathrm{cm}^{3}$ at $61.5^{\circ} \mathrm{N}$ at the peak of the profile. In the top panel on the right side of Fig. 13, a latitudinal average on the SCIAMACHY retrieval grid used in this study is provided.
We inverted the SCDs either by 1-D or 2-D approach. The resulting profiles are shown in the middle panel of Fig. 13 for 1-D case on the left and for 2-D case on the right side. The difference to the original trace gas distribution averaged on the retrieval grid used for SCIAMACHY measurements is plotted for both cases in the bottom panel. For the 2-D retrieval a much better agreement can be realized for latitudes below $\sim 68^{\circ} \mathrm{N}$ i.e. for the latitudinal regions corresponding to the 2nd, 3rd and 4th scanning sequence of SCIAMACHY. The improvement for the 2-D retrieval is up to $10 \%$ for values at the peak of the profile and it increases for altitudes below the peak.

Additionally, we modelled SCDs for a trace gas distribution with positive gradient towards the instrument (see Fig. 14). Also for this case, a stepwise gradient on a five 


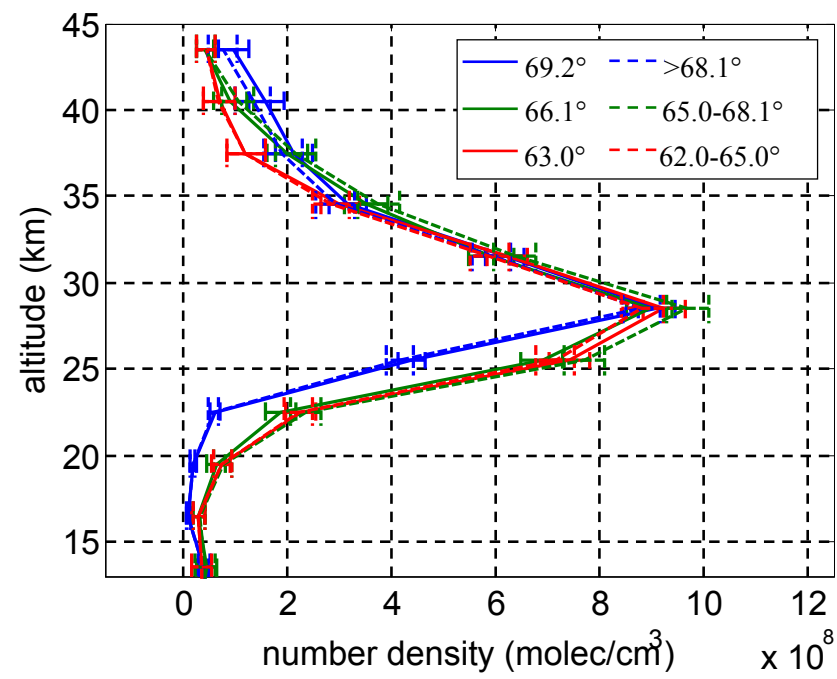

Fig. 12. Comparison between retrieved profiles using 1-D approach (solid lines) and 2-D approach (dashed lines) for orbit 15146 on 22 January 2005 for $\mathrm{NO}_{2}$ for the first three northern states of descending part of the orbit.

times finer latitudinal grid than the measurement grid of SCIAMACHY and a profile with Gaussian shape is used. The peak of the profile is at $19.5 \mathrm{~km}$ and the $\sigma$-parameter equals again $4 \mathrm{~km}$. The gradient is from $3.5 \times 10^{7} \mathrm{molec} / \mathrm{cm}^{3}$ at $71^{\circ} \mathrm{N}$ to zero at $61.5^{\circ} \mathrm{N}$ at the peak of the profile. In the top panel on the right side in Fig. 13 a latitudinal average on the SCIAMACHY retrieval grid as used in this study is provided.

The profiles, resulting either by 1-D or 2-D retrieval method are shown in the middle panel of Fig. 13 on the left and on the right side, respectively. The difference between the original trace gas distribution averaged on the retrieval grid used for SCIAMACHY measurements is plotted for both cases in the bottom panel. Also in this case, a much better agreement for the 2-D retrieval can be realized for the latitudinal regions corresponding to 2nd, 3rd and 4th scanning sequence of SCIAMACHY. For the 1-D approach a downward shift of the retrieved profile peak and overestimated values of up to $100 \%$ are observed. In contrast, the 2 $\mathrm{D}$ approach provides values being much closer to the original distribution used for the study and averaged on the retrieval grid.

To conclude, these verification studies showed that, by utilizing the overlap of consecutive limb scanning sequences, the 2-D approach allows an improved inversion of the related measurements. It has been demonstrated that this approach is correcting for the effect of horizontal gradients in limb retrievals.

\section{Conclusions}

SCIAMACHY provides scattered light measurements in limb geometry from which atmospheric trace gas profiles can be retrieved successfully. The two step approach (DOAS, RTM combined by optimal estimation) allows to investigate the effects of spectroscopy and radiative transfer on profiles separately. The assumption of horizontally homogeneous trace gas distributions can lead to errors in the retrieved profiles, in particular at locations where strong horizontal gradients occur e.g. at the edge of polar vortex. The inversion of SCDs from several consecutive SCIAMACHY limb scanning sequences simultaneously in one inversion constraint is performed by applying box AMFs resolved both in altitude and latitude. This approach allows taking into account the spatial correlation between these measurements and thereby improving the profile retrieval. This tomographic application for SCIAMACHY UV/VIS measurements is demonstrated for the first time. It allows the correction for cases with large horizontal gradients in flight/viewing direction: concentrations and profile shape are corrected in the true direction for both types of gradients (positive and negative). Such gradients can occur for several stratospheric trace gases due to their dependence on meteorological conditions, transport, solar illumination and photochemistry.

For situations with practically no horizontal gradients, no difference between the 1-D and the 2-D retrieval is observed. A better agreement of the 2-D approach with the reality was demonstrated for the verification studies, in which SCDs were modelled for the simple scenarios with either a negative or a positive gradient towards the instrument.

For the first limb scanning sequence of a SCIAMACHY orbit, where no overlap with preceding measurements exists, strong gradients in photochemically active species like $\mathrm{NO}_{2}$ or OClO can occur, especially for large SZA. A method to correct for this diurnal effect has been described by McLinden et al. (2006). Currently, we are working on an algorithm to combine this photochemical modelling with the 2-D approach presented in the article at hand. By combination of this 2-D approach and photochemical modelling, a more detailed correction for horizontal gradients will be achieved in a future modification of the retrieval algorithm.

For other parts of the SCIAMACHY orbit, where nadir scanning sequences are performed between limb scanning sequences, the presented algorithm can not be applied in its current form. It is giving only unconvincing improvement because of a poor overlap of the sensitivity areas of consecutive limb scanning sequences. This requires further studies and improvements in the algorithm. Also, in additional studies the effect of possible gradients across flight/viewing direction should be investigated because the 2-D approach presented here did not account for it.

A small distance between limb scanning sequences improves the horizontal resolution of the measurements; therefore an instrument using only UV/vis limb measurements 

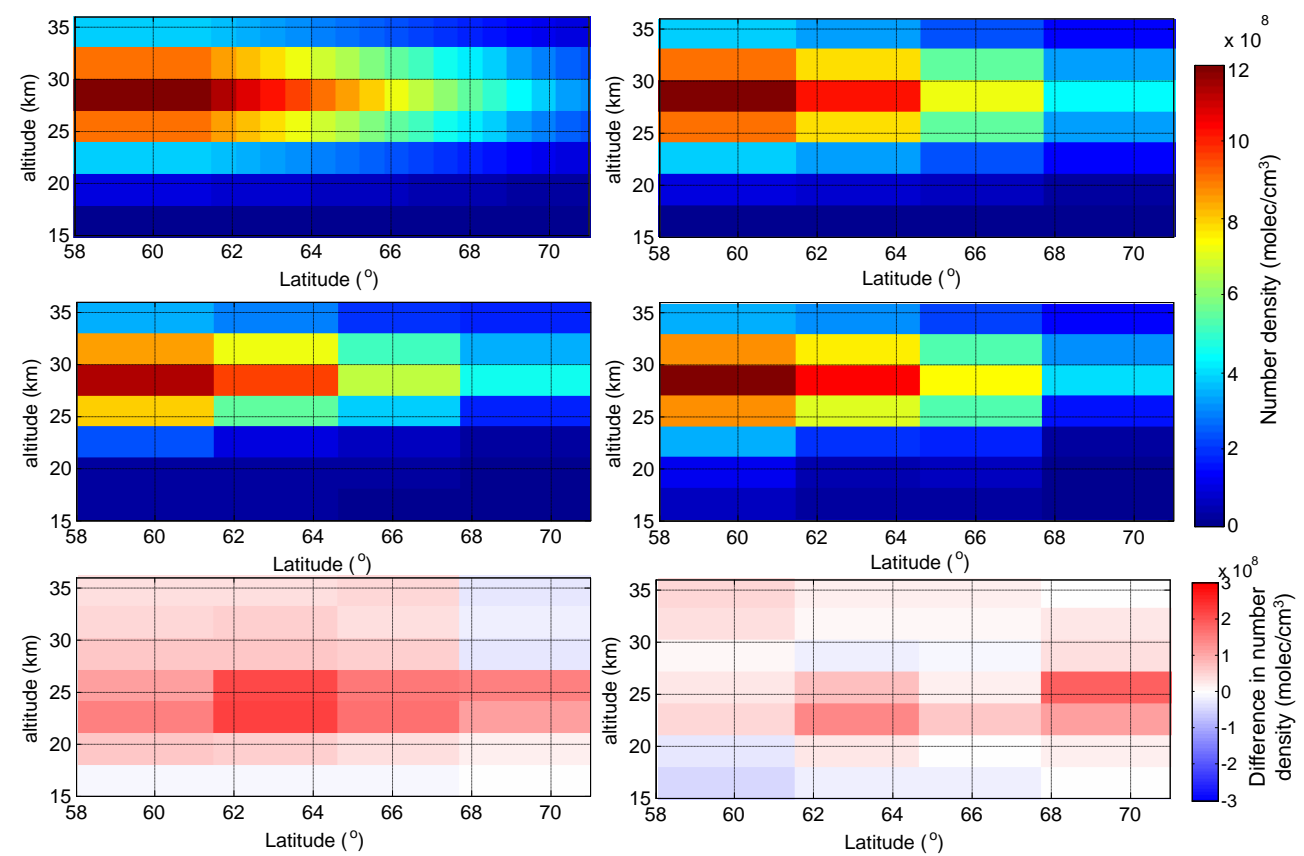

Fig. 13. Upper panel left: Sample verification profiles with negative gradient towards instrument applied for simulation of SCDs as function of latitude. Upper panel right: The same but averaged on SCIAMACHY retrieval grid. Middle panel: Retrieved profiles from simulated SCDs for 1-D case (left) and 2-D case (right). The difference between the original verification profile averaged on SCIAMACHY retrieval grid and the simulated retrievals is shown in the bottom panel for 1-D case (left) and 2-D case (right).
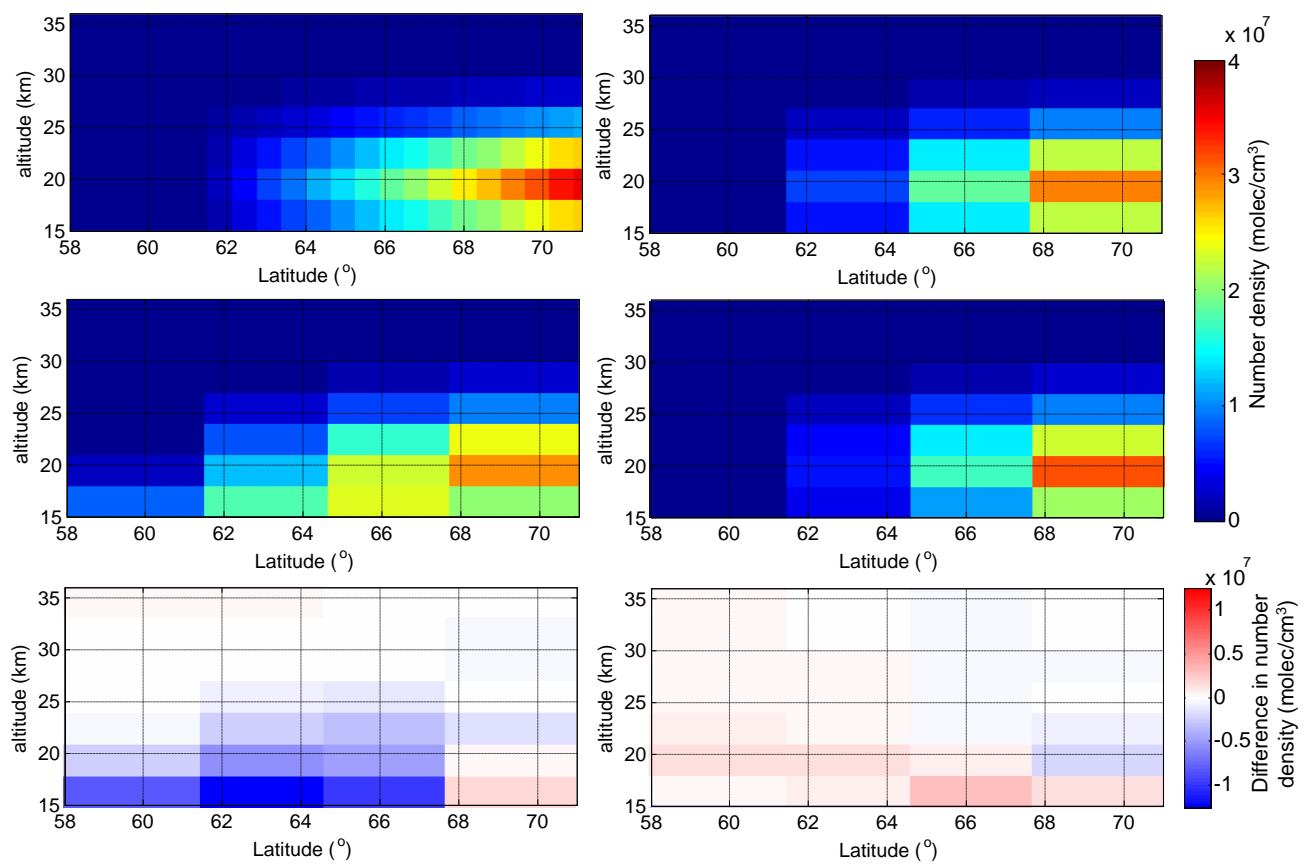

Fig. 14. Upper panel left: Sample verification profiles with positive gradient towards instrument applied for simulation of SCDs as function of latitude. Upper panel right: The same but averaged on SCIAMACHY retrieval grid. Middle panel: Retrieved profiles from simulated SCDs for 1-D case (left) and 2-D case (right). The difference between the original verification profile averaged on SCIAMACHY retrieval grid and the simulated retrievals is shown in the bottom panel for 1-D case (left) and 2-D case (right). 


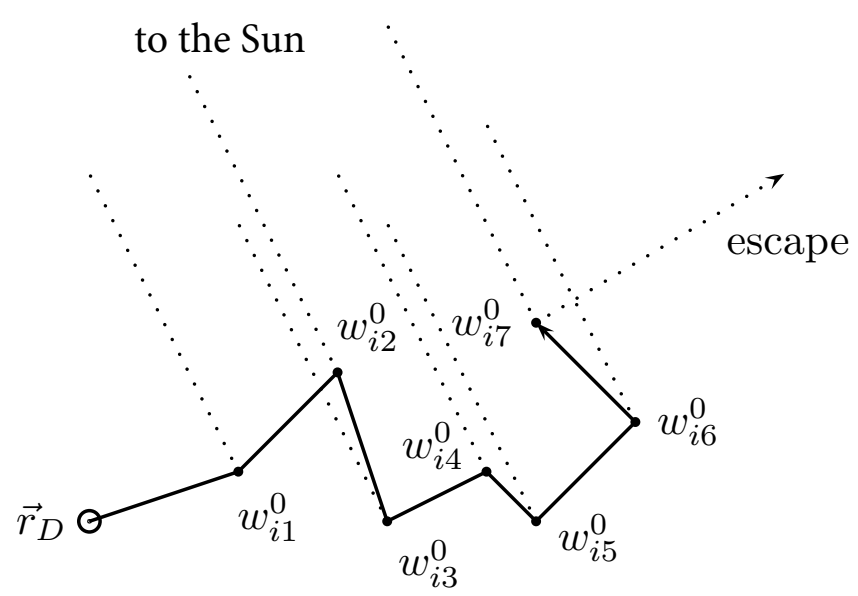

Fig. A1. Example of a backward trajectory $i$, the dotted lines lead to the sun. $\boldsymbol{r}_{D}$ is the position of the detector. $i 1 . . i 7$ are scatter events of the trajectory. They are assigned with basic weights $w_{i 1}^{0} . . w_{i 7}^{0}$ quantifying the probability for light to reach the scatter event without extinction by scattering and beeing scattered into the trajectory.

i.e. without performing nadir observations in between two limb scanning sequences would be an advantage and further improve the accuracy of the retrieved profiles.

\section{Appendix A}

\section{Calculation of box air mass factors by RTM "Tracy-II"}

For this study the backward Monte Carlo RTM "Tracy-II" calculates box AMFs according to Eq. (3) given in Sect. 3.2. We now want to describe (1) how the intensity (as measured at the instrument position) is estimated in our model and (2) how the derivative of the logarithm of the intensity is calculated, leading to the box AMFs.

In the following a path which light would take if it is emitted from the detector until it leaves the atmosphere is named a "backward trajectory" or just "trajectory". A group of backward trajectories calculated for the same RTM configuration is called "backward trajectory ensemble". Each trajectory of a backward trajectory ensemble is generated obeying the distribution densities of (1) the free path length, (2) the type of the scatter object and (3) the scatter angle (path generation). In a second step (weighting), each scatter event on a trajectory is assigned with a weight which is a measure of the probability that sun light reaches the detector.

\section{A1 Intensity}

Intensity is defined as sun normalized radiance, thus the ratio of the radiance observed by the instrument and the solar irradiance. Initially, it is calculated from the backward trajectory ensemble simulated by "Tracy-II" without considering any absorption (compare Sect. 3.3). Therefore each scatter event $j$ on a trajectory $i$ is characterized by a basic weight:

$w_{i j}^{0}=\frac{P\left(\mu_{i j}, \boldsymbol{r}_{i j}\right)}{4 \pi} \exp \left(-\int_{\boldsymbol{r}_{i j}}^{\odot} \varepsilon_{S}(\boldsymbol{r}) d r\right)$

Here, $P$ is the scattering phase function of the scatter event $(i j)$ at position $\boldsymbol{r}_{i j}$ with respect to the scatter angle $\mu_{i j}$. In the second factor $\odot$ denotes the sun and $\varepsilon_{S}(\boldsymbol{r})$ is the total scatter coefficient at position $\boldsymbol{r}$. Both factors together multiplied by the solar disc solid angle may be interpreted as the probability, that sun light reaches the scatter event $j$ and the photons are scattered into the trajectory $i$.

For a backward trajectory ensemble with $N$ trajectories the intensity without absorption is calculated by averaging the intensity estimates $I_{i}$, where each $I_{i}$ is defined as the sum of the contributions of all scatter events $j$ belonging to the backward trajectory $i$ :

$I=\lim _{N \rightarrow \infty} \frac{1}{N} \sum_{i=1}^{N} \sum_{j=1}^{m_{i}} w_{i j}^{0}$

where $m_{i}$ is the number of scatter events for the $i$ th trajectory (see Fig. A1). In theory the equal sign is valid for a large number of photons, in practice $N$ can be finite to have a certain degree of accuracy.

In order to consider absorption the basic weights $w_{i j}^{0}$ are modified as follows:

$w_{i j}=w_{i j}^{0} \cdot \exp \left(-\int_{D}^{\odot} \beta_{i j} \beta_{\text {total }}(\boldsymbol{r}) d r\right) \cdot A_{i j}$

with absorption coefficient $\beta_{\text {total }}$ of all absorbers at position $r$ :

$\beta_{\text {total }}(\boldsymbol{r})=\sum_{t}^{\substack{\text { tracegases } \\ n_{t}}}(\boldsymbol{r}) \sigma_{t}(\boldsymbol{r})=\sum_{t}^{\text {tracegases }} \beta_{t}(\boldsymbol{r})$

The basic weight $w_{i j}^{0}$ is reduced by a Lambert-Beer-like factor and $A_{i j}$ which is the product of all aerosol single scattering albedos and ground albedos on the backward trajectory $i$ from the detector until the scatter event $j$. In case of Rayleigh scattering events the albedo is set to one. Equation (A3) may be interpreted as a measure of the probability of light to reach the detector $D$ through the trajectory $i$ by scatter event $j$, considering absorption on condition that the whole backward trajectory ensemble is representative for a physical photon ensemble emerging from the detector. For a sampled backward trajectory this is always true, because every backward trajectory was generated obeying the probability distributions of the free path length, the scatter object and the angle of scattering. For details on the theory behind the method the interested reader is referred to Postylyakov (2004) or Marchuk et al. (1980). 
Finally, the formula for the intensity with absorption $I_{g}$ is the same as Eq. (A2) but with $w_{i j}$ instead of $w_{i j}^{0}$ :

$I_{g}=\lim _{N \rightarrow \infty} \frac{1}{N} \sum_{i=1}^{N} \sum_{j=1}^{m_{i}} w_{i j}$

Please note that "Tracy-II" calculates Sun normalized radiance. Because in terms of Eq. (2) only a ratio of intensities is relevant, we also refer to $I_{g}$ for reasons of consistency here.

A2 Logarithmic derivatives of the intensity and box air mass factors

For the calculation of the derivative of logarithm of the intensity and later box AMFs one has to investigate the LambertBeer-like term in Eq. (A3) only, because all other factors do not depend on the tracegas profile. The derivative of this expression with respect to the absorption coefficient $\beta_{t b}$ of one tracegas $t$ in the box $b$ is:

$$
\frac{d}{d \beta_{t b}} w_{i j}=-l_{i j}^{b} w_{i j}
$$

where $l_{i j}^{b}$ is the light path length associated with trajectory $i$ and the scatter event $j$ in the box $b$. Taking this into account and applying the chain rule, the logarithmic derivative of the intensity in Eq. (A5) with respect to the absorption coefficient $\beta_{t b}$ of one tracegas $t$ (that of the interest) in box $b$ becomes:

$\frac{d}{d \beta_{t b}} \log \left(I_{g}\right)=-\frac{\sum_{i=1}^{N} \sum_{j=1}^{m_{i}} l_{i j}^{b} w_{i j}}{\sum_{i=1}^{N} \sum_{j=1}^{m_{i}} w_{i j}}=-\left\langle L_{b}\right\rangle$

where $\left\langle L_{b}\right\rangle$ is the weighted average of trajectory lengths through the box with their weighting factors. Please note that in Eq. (A7) also trajectories not crossing the box are considered: Their length through the box is zero but their weight still contributes to the sum in the denominator.

It turns out, that Eq. (A7) is equal for any trace gas considered in a certain atmospheric scenario, because the derivative in Eq. (A6) is the same for all absorbers, i.e. it depends on (not necessarily small) optical depth only.

Inserting Eq. (A7) in Eq. (3), the elements $A M F_{g b}$ of the AMF matrix in Eq. 1 with respect to the box $b$ and the geometry $g$ finally are:

$A M F_{g b}=\frac{\left\langle L_{b}\right\rangle}{h_{b}}$

Acknowledgements. We want to thank ESA for providing data of SCIAMACHY, DLR for providing the SCIAMACHY geolocation maps via SOST webpage (http://atmos.caf.dlr.de/projects/scops/), NILU and ECMWF for meteorological data, University of Latvia and ESF for support. One author (S. Kühl) is funded by the DFG (Deutsche Forschungs Gemeinschaft). We thank Claudia Keller (MPI for Chemistry, Mainz) for proofreading of the manuscript.

Edited by: A. Richter

\section{References}

Bhartia, P. K., McPeters, R. D., Mateer, C. L., Flynn, L. E. and Wellemeyer, C.: Algorithm for the estimation of vertical ozone profiles from the backscattered ultraviolet technique, J. Geophys. Res., 101, 18, 793-18,806, 1996.

Bovensmann H., Burrows, J. P., Buchwitz, M., Frerick, J., Noël, S., Rozanov, V. V., Chance, K. V., and Goede, A. P. H.: SCIAMACHY: Mission objectives and measurement modes, J. Atmos. Sci., 56, 127-150, 1999.

Brühl, C. and Crutzen, P. J.: MPIC two-dimensional model, in: The Atmospheric Effect of Stratospheric Aircraft, 1292, edited by: Prather, M. J. and Remsberg, E. E., NASA Ref. Publications, 103-104, 1993.

Butz, A., Bösch, H., Camy-Peyret, C., Chipperfield, M., Dorf, M., Dufour, G., Grunow, K., Jeseck, P., Kühl, S., Payan, S., Pepin, I., Puķīe, J., Rozanov, A., von Savigny, C., Sioris, C., Wagner, T., Weidner, F., and Pfeilsticker, K.: Inter-comparison of stratospheric $\mathrm{O}_{3}$ and $\mathrm{NO}_{2}$ abundances retrieved from balloon borne direct sun observations and Envisat/SCIAMACHY limb measurements, Atmos. Chem. Phys., 6, 1293-1314, 2006, http://www.atmos-chem-phys.net/6/1293/2006/.

Carlotti, M., Brizzi, G., Papandrea, E., Prevedelli, M., Ridolfi, M., Dinelli, B. M., and Magnani, L.: GMTR: Two-dimensional geofit multitarget retrieval model for Michelson Interferometer for Passive Atmospheric Sounding/Environmental Satellite observations, Appl. Optics, 45, 716-727, 2006.

Carlotti, M., Dinelli, B. M., Raspollini, P., and Ridolfi, M.: Geo-fit Approach to the Analysis of Limb-Scanning Satellite Measurements, Appl. Optics, 40, 1872-1885, 2001.

Degenstein, D. A., Llewellyn, E. J. and Lloyd, N. D.: Volume Emission Rate Tomography from a Satellite Platform, Appl. Optics, 42, 1441-1450, 2003.

Deutschmann, T. and Wagner, T.: TRACY-II Users manual, University of Heidelberg, Heidelberg, Germany, available at: http://joseba.mpch-mainz.mpg.de/tdeutschmann/tracy_ II/download/index.html, 2007

Dorf M., Bösch, H., Butz, A., Camy-Peyret, C., Chipperfield, M. P., Engel, A., Goutail, F., Grunow, K., Hendrick, F., Hrechanyy, S., Naujokat, B., Pommereau, J.-P., Van Roozendael, M., Sioris, C., Stroh, F., Weidner, F., and Pfeilsticker, K.: Balloon-borne stratospheric BrO measurements: Comparison with Envisat/SCIAMACHY BrO limb profiles, Atmos. Chem. Phys., 6, 2483-2501, 2006, http://www.atmos-chemphys.net/6/2483/2006/.

European Centre for Medium-Range Weather Forecasts (ECMWF): Temperature and potential vorticity analyses, Dan. Meteorol. Inst., Copenhagen, 2000.

Haley, C. S., Brohede, S. M., Sioris, C. E., Griffioen, E., Murtagh, D. P., McDade, I. C., Eriksson, P., Llewellyn, E. J., Bazureau, A., and Goutail, F.: Retrieval of stratospheric $\mathrm{O}_{3}$ and $\mathrm{NO}_{3}$ profiles from Odin Optical Spectrograph and Infrared Imager System (OSIRIS) limb-scattered sunlight measurements, J. Geophys. Res., 109, D16303, doi:10.1029/2004JD004588, 2004.

Hoogen, R., Rozanov, V. V. and Burrows, J. P.: Ozone profiles from GOME satellite data: Algorithm description and first validation, J. Geophys. Res., 104, 8263-8280, 1999.

Krecl, P., Haley, C. S., Stegman, J., Brohede, S. M., and Berthet G.: Retrieving the vertical distribution of stratospheric OClO from Odin/OSIRIS limb-scattered sunlight measurements, At- 
mos. Chem. Phys., 6, 1879-1894, 2006,

http://www.atmos-chem-phys.net/6/1879/2006/.

Kühl, S.: Quantifying Stratospheric chlorine chemistry by the satellite spectrometers GOME and SCIAMACHY, PhD Thesis, Universität Heidelberg, Heidelberg, Germany, 177 pp., available at: http://www.ub.uni-heidelberg.de/archiv/5664/, 2005.

Kühl, S., Puķīe, J., Deutschmann, T., Platt, U., and Wagner, T.: SCIAMACHY Limb Measurements of $\mathrm{NO}_{2}, \mathrm{BrO}$ and $\mathrm{OClO}$, Retrieval of vertical profiles: Algorithm, first results, sensitivity and comparison studies, in Press, doi:10.1016/j.asr.2007.10.022, Adv. Space Res., 2007.

Llewellyn E. J., Lloyd, N. D., Degenstein, D. A., Gattinger, R. L., Petelina, S. V., Bourassa, A. E., Wiensz, J. T., Ivanov, E. V., McDade, I. C., Solheim, B. H., McConnell, J. C., Haley, C. S., von Savigny, C., Sioris, C. E., McLinden, C. A., Griffioen, E., Kaminski, J., Evans, W. F. J., Puckrin, E., Strong, K., Wehrle, V., Hum, R. H., Kendall, D. J. W., Matsushita, J., Murtagh, D. P., Brohede, S., Stegman, J., Witt, G., Barnes, G., Payne, W. F., Piché, L., Smith, K., Warshaw, G., Deslauniers, D.-L., Marchand, P., Richardson, E. H., King, R. A., Wevers, I., McCreath, W., Kyrölä, E., Oikarinen, L., Leppelmeier, G. W., Auvinen, H., Mégie, G., Hauchecorne, A., Lefèvre, F., de La Nöe, J., Ricaud, P., Frisk, U., Sjoberg, F., von Schéele, F., and Nordh, L.: The OSIRIS instrument on the Odin spacecraft, Can. J. Phys., 82, 411-422, 2004.

Loughman, R. P., Griffioen, E., Oikarinen, L., Postylyakov, O. V., Rozanov, A., Flittner, D. E., and Rault, D. F.: Comparison of radiative transfer models for limb-viewing scattered sunlight measurements, J. Geophys. Res., 109, D06303, doi:10.1029/2003JD003854, 2004.

Marchuk, G. I., Mikhailov, G. A., Nazaraliev, M. A., Darbinyan, R. A., Kargin, B. A., and Elepov, B. S.: The Monte Carlo methods in atmospheric optics, Springer Verlag, New York, 208 pp., 1980.

Davis, A. B. and Knyazikhin, Y.: A primer in three-dimensional radiative transfer, in: Marshak, A., Davis, A. B.: 3D Radiative Transfer in Cloudy Atmospheres, Springer Verlag, New York, 686 pp., 2005.

Menke, W.: Geophysical data analysis: discrete inverse theory, Academic Press, 289 pp., 1999.

McLinden, C. A., Haley, C. S., and Sioris, C. E.: Diurnal effects in limb scatter observations, J. Geophys. Res., 111, D14302, doi:10.1029/2005JD006628, 2006.

Natarajan, M., Deaver, L. E., Thompson, E., and Magill, B.: Impact of twilight gradients on the retrieval of mesospheric ozone from HALOE, J. Geophys. Res., 110, D13305, doi:10.1029/2004JD005719, 2005.

Oikarinen, L., Sivhola, E., and Kyrölä, E.: Multiple-scattering radiance in limb-viewing geometry, J. Geophys. Res., 104, 31 261$31274,1999$.

Postylyakov, O. V.: Linearized vector radiative transfer model $\mathrm{MCC}++$ for a spherical atmosphere. J. Quant. Spectrosc. Radiat. Transfer, 88, 297-317, doi:10.1016/j.jqsrt.2004.01.009, 2004.
Puķīte, J., Kühl, S., Deutschmann, T., Wilms-Grabe, W., Friedeburg, C., Platt, U., and Wagner, T.: Retrieval of stratospheric trace gases from SCIAMACHY limb measurements, Proceedings of the First Atmospheric Science Conference, 812 May, ESA/ESRIN, Frascati, Italy, ESA SP-628, available at: http://earth.esa.int/workshops/atmos2006/participants/1148/ paper_proc_Frasc_2.pdf, 2006.

Rault D. F.: Ozone profile retrieval from Stratospheric Aerosol and Gas Experiment (SAGE III) limb scatter measurements, J. Geophys. Res., 110, D09309, doi:10.1029/2004JD004970, 2005.

Ridolfi, M., Magnani, L., Carlotti, M., and Dinelli, B. M.: MIPASENVISAT Limb-Sounding Measurements: Trade-Off Study for Improvement of Horizontal Resolution, Appl. Optics, 43, 5814 5824, 2004.

Rodgers, C. D.: Inverse methods for atmospheric sounding. Theory and practice, World Scientific Publishing Co. Ltd., Singapore, 238 pp., 2000.

Rozanov, A., Bovensmann, H., Bracher, A., Hrechanyy, S., Rozanov, V., Sinnhuber, M., Stroh, F., and Burrows, J. P.: $\mathrm{NO}_{2}$ and $\mathrm{BrO}$ vertical profile retrieval from SCIAMACHY limb measurements: sensitivity studies, Adv. Space Res., 36, 846-854., 2005.

Sinnhuber, B.-M., Rozanov, A., Sheode, N., Afe, O. T., Richter, A., Sinnhuber, M., Wittrock, F., and Burrows, J. P.: Global observations of stratospheric bromine monoxide from SCIAMACHY, Geophys. Res. Lett., 32, L20810, 2005.

Sioris, C. E., Kovalenko, L. J., McLinden, C. A., Salawitch, R. J., Van Roozendael, M., Goutail, F., Dorf, M. Pfeilsticker, K., Chance, K., von Savigny, C., Liu, X., Kurosu, T. P., Pommereau, J.-P., Bösch, H., and Frerick, J.: Latitudinal and vertical distribution of bromine monoxide in the lower stratosphere from Scanning Imaging Absorption Spectrometer for Atmospheric Chartography limb scattering measurements, J. Geophys. Res., 111, D14301, doi:10.1029/2005JD006479, 2006.

Sioris, C. E., Kurosu, T. P., Martin, R. V., and Chance, K.: Stratospheric and tropospheric $\mathrm{NO}_{2}$ observed by SCIAMACHY: First results, Adv. Space Res., 34, 780-785, 2004.

von Savigny, C., Rozanov, Bovensmann, A. H., Eichmann, K.-U., Noël, S., Rozanov, V. V., Sinnhuber, B.-M., Weber, M. and Burrows, J. P.: The ozone hole breakup in September 2002 as seen by SCIAMACHY on ENVISAT, J. Atmos. Sci., 62, 721-734, 2005.

Wagner, T., Burrows, J. P., Deutschmann, T., Dix, B., von Friedeburg, C., Frieß, U., Hendrick, F., Heue, K.-P., Irie, H., Iwabuchi, H., Kanaya, Y., Keller, J., McLinden, C. A., Oetjen, H., Palazzi, E., Petritoli, A., Platt, U., Postylyakov, O., Puksīe, J., Richter, A., van Roozendael, M., Rozanov, A., Rozanov, V., Sinreich, R., Sanghavi, S., and Wittrock, F.: Comparison of Box-AirMass-Factors and Radiances for Multiple-Axis Differential Optical Absorption Spectroscopy (MAX-DOAS) Geometries calculated from different UV/visible Radiative Transfer Models, Atmos. Chem. Phys., 7, 1809-1833, 2007, http://www.atmos-chem-phys.net/7/1809/2007/. 